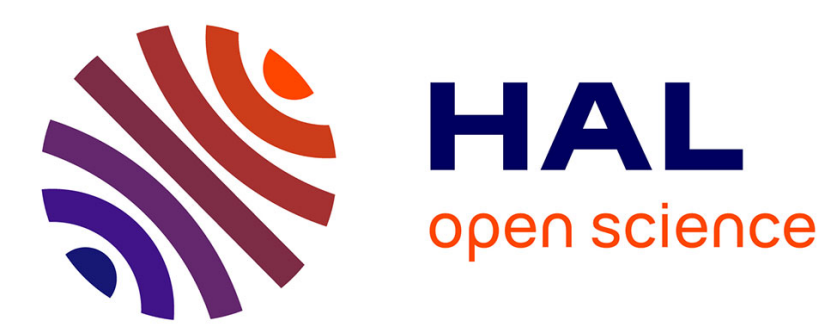

\title{
Pistes de réflexion pour l'évaluation et le financement des interventions complexes en santé publique
}

Cécile Marie Dupin, Eric Breton, Joëlle Kivits, Laetitia Minary

\section{To cite this version:}

Cécile Marie Dupin, Eric Breton, Joëlle Kivits, Laetitia Minary. Pistes de réflexion pour l'évaluation et le financement des interventions complexes en santé publique. Santé Publique, 2015, 5, pp.653-657. 10.3917/spub.155.0653 . hal-01573908

\section{HAL Id: hal-01573908 \\ https://hal.univ-lorraine.fr/hal-01573908}

Submitted on 10 Aug 2017

HAL is a multi-disciplinary open access archive for the deposit and dissemination of scientific research documents, whether they are published or not. The documents may come from teaching and research institutions in France or abroad, or from public or private research centers.
L'archive ouverte pluridisciplinaire HAL, est destinée au dépôt et à la diffusion de documents scientifiques de niveau recherche, publiés ou non, émanant des établissements d'enseignement et de recherche français ou étrangers, des laboratoires publics ou privés. 


\section{PISTES DE RÉFLEXION POUR L'ÉVALUATION ET LE FINANCEMENT DES INTERVENTIONS COMPLEXES EN SANTÉ PUBLIQUE}

Cécile Marie Dupin, Éric Breton, Joëlle Kivits, Laetitia Minary

S.F.S.P. $\mid$ « Santé Publique »

2015/5 Vol. 27 | pages 653 à 657

ISSN 0995-3914

Article disponible en ligne à l'adresse :

http://www.cairn.info/revue-sante-publique-2015-5-page-653.htm

!Pour citer cet article :

Cécile Marie Dupin et al., « Pistes de réflexion pour l'évaluation et le financement des interventions complexes en santé publique », Santé Publique 2015/5 (Vol. 27), p. 653-657.

Distribution électronique Cairn.info pour S.F.S.P..

(C) S.F.S.P.. Tous droits réservés pour tous pays.

La reproduction ou représentation de cet article, notamment par photocopie, n'est autorisée que dans les limites des conditions générales d'utilisation du site ou, le cas échéant, des conditions générales de la licence souscrite par votre établissement. Toute autre reproduction ou représentation, en tout ou partie, sous quelque forme et de quelque manière que ce soit, est interdite sauf accord préalable et écrit de l'éditeur, en dehors des cas prévus par la législation en vigueur en France. Il est précisé que son stockage dans une base de données est également interdit. 


\section{Pistes de réflexion pour l'évaluation et le financement des interventions complexes en santé publique}

\section{Reflections on the evaluation and funding of complex public health interventions}

Cécile Marie Dupin ${ }^{1,2}$, Eric Breton ${ }^{1,2}$, Joëlle Kivits, ${ }^{3,4,5}$, Laetitia Minary ${ }^{3,6}$ et le groupe

de réflexion sur l'évaluation et le financement des interventions complexes en santé publique ${ }^{7}$

\section{$\hookrightarrow$ Résumé}

En France, dans un contexte grandissant d'inégalités sociales de santé, la nécessité d'actions sur l'environnement et sur les déterminants sociaux de la santé (DSS) appelle la recherche en promotion de la santé à contribuer à l'avancement des connaissances dans ce domaine. Or, l'enjeu est non seulement de concevoir et d'élaborer des interventions adaptées aux contextes, actives sur les déterminants de santé ciblés, mais aussi de construire des dispositifs d'évaluation de ces interventions dites complexes. Un groupe de chercheurs et de représentants des institutions finançant la recherche s'est réuni le 2 juin 2014 à Paris pour réfléchir aux expériences en cours en France. Cinq pistes de réflexion issues de ces échanges ont été formulées : (i) le contexte de l'intervention doit être considéré comme ingrédient actif, (ii) l'évaluation des actions doit être guidée par une logique d'intervention rigoureuse, (iii) les essais contrôlés randomisés, peu adaptés à l'évaluation en milieu complexe, doivent être accompagnés de modèles alternatifs d'évaluation, notamment l'analyse de processus de l'intervention, (iv) la recherche interventionnelle doit être collaborative voire co-construite, (v) la formation en santé publique doit faire une large place à la pluralité des approches et méthodes d'évaluation. Les pistes de réflexions présentées ici, issues d'un état des lieux du contexte français, soulignent que face à ces défis, financeurs et acteurs français de la recherche doivent s'engager dans des concertations en termes de recherche interventionnelle, d'évaluation et d'implémentation des interventions complexes en santé publique.

Mots-clés : Déterminants sociaux de la santé ; Essais contrôlés randomisés comme sujet; Évaluation; Promotion de la santé ; Recherche participative communautaire.

\begin{abstract}
$[$ Summary
In France, in a context of growing health inequalities, the need for action on life settings and, more broadly, on the social determinants of health (SDH), requires a contribution from health promotion research. Today's challenge is not only to design interventions tailored to contexts and actively targeting SDH, but also to develop innovative evaluation strategies of these complex interventions. A group of researchers and representatives from funding agencies met in Paris on June 2nd, 2014 to discuss current experiences conducted in France. The debates yielded five conclusions: (i) the context of the intervention must be considered as one of its active ingredients, (ii) evaluation must be guided by a sound intervention logic (iii) randomized controlled trials cannot capture the complexity of the environment and evaluation must be designed using alternative models, including process evaluation, (iv) interventional research should be collaborative, or co-constructed, (v) public health training should cover the diversity of evaluative methods. The conclusions described here, in the context of France, stress that to address these challenges, funding agencies, researchers and stakeholders should further engage in discussions concerning the conduct of interventional research, evaluation and implementation of complex public health interventions.
\end{abstract}

Keywords: Social determinants of health; Randomized controlled trials as topic; Evaluation; Health promotion; Community-based participatory research.

\footnotetext{
${ }^{1}$ Chaire Inpes « Promotion de la santé » - École des Hautes Études en santé publique - Avenue du Professeur Léon Bernard - F-35000 Rennes.

${ }^{2}$ CNRS - UMR CRAPE 6051.

${ }^{3}$ Université de Lorraine - Faculté de Médecine - École de Santé Publique - Nancy F-54000 - France.

${ }^{4}$ Université de Lorraine - Université Paris Descartes - Apemac - EA4360 - Nancy F-54000 - France.

${ }^{5}$ CHU Nancy / Pôle QSP2 - Épidémiologie et Évaluation Cliniques - Nancy F-54000 - France.

${ }^{6}$ École de santé publique de l'Université de Montréal - Montréal - Québec - Canada.

${ }^{7}$ Le groupe de réflexion sur l'évaluation et le financement des interventions complexes en santé publique est composé de : Linda Cambon (Agence régionale de santé de Picardie); Eric Breton, Cécile Dupin, Françoise Jabot, Jocelyn Raude, William Sherlaw (École des Hautes Études en Santé Publique) ; Jérome-Philippe Garsi (Fondation MGEN pour la santé publique) ; Pierre Arwidson, Virginie Simond (Inpes) ; Bastien Affeltranger (Institut National du Cancer) ; Maitena Milhet (Observatoire Français des Drogues et Toxicomanies) ; Julie Pironom, Frank Pizon, Cédric Kempf (Laboratoire ACTé EA4281 ESPE Clermont-Auvergne, Université Blaise Pascal) ; François Alla, Joëlle Kivits, Laëtitia Minary, Justine Trompette (EA 4360 APEMAC, Université de Lorraine) ; Marie-Hélène Metzger (CHU-Université Lyon).
} 


\section{Introduction}

Le contexte français, notamment marqué par des inégalités en santé grandissantes, nécessite la mise en œuvre d'actions sur l'environnement et sur les déterminants sociaux de la santé (DSS) [1], par des actions intersectorielles (transversales) et la conception de programmes participatifs [2]. Bien qu'encore insuffisamment développée en France, la recherche en prévention et promotion de la santé ambitionne de relever ces défis par le biais de la recherche interventionnelle (RI). La RI produit des connaissances sur les processus, les mécanismes, les effets et les impacts des interventions dans une visée d'utilité sociale [3]. Ce domaine contribue à l'avancement des connaissances sur les modes d'actions prometteurs, sur les déterminants sociaux de la santé [4], et sur le développement de stratégies pour leur évaluation [5].

Dites complexes en raison (i) du nombre de leurs composantes et de leurs interactions, (ii) des groupes ou niveaux organisationnels ciblés, (iii) de la variabilité des résultats, (iv) de la difficulté des comportements à adopter par les bénéficiaires et les acteurs [6], mais également du fait du système complexe dans lesquelles elles sont implantées, les interventions en promotion de la santé exigent des démarches évaluatives innovantes [5, 7]. Dans le cadre d'actions sur les milieux de vie, les environnements, ou encore les changements de comportements, il ne s'agit en effet plus uniquement de répondre à la question : «Est-ce que l'intervention fonctionne? » mais d'envisager les questions : «Comment fonctionne-t-elle? ", «Auprès de quelle(s) population(s) ? » et « Pourquoi ? ». L'enjeu de l'évaluation de telles interventions complexes est non seulement de concevoir et d'élaborer des interventions adaptées aux contextes de vie et d'action, agissant au mieux sur les déterminants de santé ciblés par l'intervention, mais également d'envisager les effets de ces interventions dans des contextes différents $[8,9]$.

Ainsi est-il observé un recours de plus en plus fréquent aux recommandations du Medical Research Council (MRC) [5]. Le cadre proposé par le MRC permet de pallier les limites du modèle de l'essai contrôlé randomisé (ECR) individuel, peu adapté à la problématique de l'évaluation des interventions complexes [9]. S'il permet de répondre à la question de l'efficacité théorique d'une intervention, il ne fournit pas d'information sur les mécanismes de cette intervention et ne permet donc pas d'aborder les éléments de complexité tels que le contexte de l'intervention, les dimensions organisationnelles, populationnelles ou encore environnementales de l'intervention. Par ailleurs, les méthodologies utilisées à des fins évaluatives doivent contribuer, selon Trickett [10], à l'acceptabilité, la pérennisation, et la transférabilité des interventions innovantes mises en œuvre.

Cette orientation scientifique devenue indispensable pour soutenir une pratique de santé publique basée sur les données probantes est celle de la recherche interventionnelle en santé des populations (RI). La RI, alimentée par la recherche dans de nombreux domaines et disciplines, se définit comme «l'utilisation de méthodes scientifiques pour produire des connaissances concernant les interventions et les programmes afin d'avoir un impact favorable sur les DSS » [3]. La RI diffère ainsi de la recherche évaluative qui peut être conduite indépendamment de la construction des interventions. Le point focal est concentré sur le « comment » du fonctionnement des interventions [3]. Au-delà de la production de connaissances sur l'efficacité des interventions, la RI favorise la compréhension des facteurs influençant ces interventions, leurs mécanismes d'actions et les interactions entre contexte, intervention et résultats en mobilisant des méthodes d'évaluation qui doiventêtre adaptées à la complexité des interventions [11]. Pour McLaren et Hawe [12], la RI doit reposer sur une perspective socioécologique sous-tendant une interaction entre les différents niveaux d'une intervention (personnel, organisationnel, communautaire, politique). La recherche interventionnelle représente donc une source de savoirs riches pour l'implantation d'actions et la prise de décision en santé publique [13].

Face à ces constats, il s'agit pour les décideurs de soutenir le développement d'actions expérimentales, d'études et de recherches en promotion de la santé qui permettent la production de connaissances sur les leviers d'action efficaces et l'évaluation de leur transférabilité dans différents contextes d'intervention [9].

\section{Pistes de réflexion pour l'évaluation et le financement d'interventions complexes en santé publique en France}

Un groupe de chercheurs et de représentants des institutions finançant la recherche s'est réuni le 2 juin 2014 à Paris pour réfléchir aux expériences en cours en France. De ces discussions, cinq pistes de réflexions ont été formulées (tableau I).

\section{Considérer le contexte comme ingrédient actif de l'intervention}

Parmi les principes qui doivent guider l'action, il est apparu primordial de reconsidérer l'importance du 
Tableau I : Cinq pistes de réflexions issues des échanges

\section{- Le contexte de l'intervention doit être considéré comme ingrédient actif.}

- La planification, le déploiement et l'évaluation des actions doivent être guidés par une logique d'intervention rigoureuse.

- Les ECR, peu adaptés à l'évaluation en milieu complexe, doivent être accompagnés de modèles alternatifs d'évaluation, notamment l'analyse de processus de l'intervention par des études qualitatives et/ou mixtes.

- Dans ces recherches interventionnelles en co-construction, l'évaluation doit être perçue comme un mode d'apprentissage.

- La formation en santé publique doit faire une large place à la pluralité des approches et méthodes d'évaluation.

Encadré 1: Une intervention intersectorielle construite sur un territoire : le programme "Ensemble la santé pour tous en Pays de Redon-Bretagne Sud"

Le programme "Ensemble la santé pour tous en Pays de Redon" (PR-BS) est une recherche interventionnelle dont l'objectif est de contribuer à l'amélioration des connaissances et des stratégies populationnelles visant l'action sur les déterminants sociaux de la santé (DSS) en région Bretagne. Ce programme, ambitionnant de participer à la réduction des inégalités sociales et territoriales de santé (ISTS) dans le Pays de Redon, rassemble un comité d'acteurs locaux intersectoriels visés par l'intervention pour une meilleure prise en compte des DSS dans les pratiques du territoire.

contexte de développement et d'évaluation des interventions complexes en santé des populations. Plus précisément, le contexte dans lequel sont déployées les interventions doit être abordé comme un élément majeur, jouant le rôle d'un ingrédient actif de l'intervention. Ce contexte comporte plusieurs niveaux : les caractéristiques et capacités des acteurs et des bénéficiaires, leurs relations interpersonnelles, le contexte territorial, organisationnel ou institutionnel, le contexte plus large (national ou international) [14]. Ainsi, les dispositifs d'intervention doivent avoir pour ambition d'être construits et implantés sur les territoires, avec les acteurs clefs, de manière à devenir effectifs en routine, et pas uniquement dans des conditions expérimentales. D'autre part, dans le cadre de l'action sur les milieux de vie, il est nécessaire de développer des partenariats entre chercheurs, intervenants et acteurs locaux qui doivent traverser les secteurs de la santé, l'éducation, la protection sociale, l'environnement, des transports et impliquer des organismes publics, privés et non gouvernementaux. Cette volonté intersectorielle est une plus-value qui doit être valorisée dès la conception des interventions (Encadré 1). La prise en compte du contexte lors de l'implantation d'une intervention et lors de son évaluation doit non seulement permettre à l'intervention de fonctionner dans un cadre réel de manière pérenne, mais également d'être transférée dans un autre contexte.

\section{S'appuyer sur une logique d'intervention rigoureuse pour planifier, expliquer et évaluer les interventions}

Le développement, la mise en œuvre et l'évaluation d'interventions complexes, nécessitent de décrire la logique liant l'action aux effets attendus et observés (Encadré 2). L'explicitation des changements visés par l'intervention doit reposer sur la formulation de théories de programmes et modèles logiques décrivant les mécanismes d'action de l'intervention. La logique d'intervention constitue ainsi une ressource qui guide l'action, mais qui permet aussi de tirer des apprentissages des interventions mises en œuvre et de capitaliser sur les expériences [15]. Elle permet de rendre compte de la chaîne causale mobilisée, générative, non linéaire et complexe, afin de décrire «ce qui fonctionne, pour qui, pour quoi et dans quelles circonstances? ? [16] et favoriser la transférabilité de l'intervention.

Encadré 2 : Une intervention complexe qui souligne l'importance de la chaîne causale : Étude Tabado

Évaluer l'efficacité d'un programme d'aide au sevrage tabagique auprès d'adolescents scolarisés en Centre de Formation des Apprentis par une étude prospective de type quasi expérimental: l'étude TABADO. Ce programme a suggéré un "effet groupe" dans le processus de sevrage tabagique: par quel processus agirait ce facteur social ?

- S'agit-il d'un effet direct d'un individu à un autre (l'entrée dans une démarche de sevrage sert d'exemple à l'entourage) ?

- Ou d'un effet indirect induit par un événement vécu ensemble (action de prévention TABADO) expérimental ?

Les ECR, peu adaptés à l'évaluation en milieu complexe, doivent être accompagnés de modèles alternatifs d'évaluation, notamment l'analyse de processus de l'intervention

Lors des échanges, il est apparu essentiel de reconsidérer la conception hiérarchique de la preuve scientifique posant les essais contrôlés randomisés (ECR) comme la référence [9]. Compte tenu de la nécessité d'adaptation des interventions à la vie réelle, et comme leur effet n'est pas séparable du contexte, il s'agit donc de privilégier l'évaluation de l'« effectiveness » d'une intervention, c'est-à-dire l'efficacité en routine $[17,18]$ plutôt que son "efficacy», c'est-à-dire l'efficacité en condition expérimentale. Le défi est en effet de construire les interventions dans les milieux, dans les environnements existants, et d'élaborer des interventions 
adaptées aux contextes. Face à cette complexité, le développement et la valorisation des modèles adaptés ou alternatifs d'évaluation doivent intégrer notamment l'évaluation de processus de l'intervention [5]. La plupart de ces modèles reposent sur une approche méthodologique mixte associant approches quantitatives et qualitatives.

\section{Dans ces recherches interventionnelles en co-construction, l'évaluation doit être perçue comme un mode d'apprentissage}

La recherche interventionnelle en santé publique est complexe, lourde, exigeante en énergie, en ressources et en compétences. Les partenariats favorisent la mise en œuvre du dispositif d'intervention et son évaluation, qui reposent alors sur une collaboration étroite entre chercheurs de plusieurs disciplines, et entre chercheurs et acteurs. En retour, ces collaborations et partenariats s'en trouvent favorisés et consolidés. La translation des données entre ces deux groupes dans le processus de développement de la preuve renforce l'intervention par les résultats de l'évaluation, et la recherche par l'intégration de la vision des acteurs dans les données produites. Ultimement, l'évaluation doit permettre d'améliorer l'intervention en l'adaptant suivant les résultats de l'évaluation, et en la confrontant à la vision des acteurs.

\section{Se former à la diversité des méthodes évaluatives}

Complémentairement à ces réflexions, le développement et la mise en œuvre de recherches interventionnelles socioécologiques innovantes doivent être soutenus par un processus d'évaluation utilisant des méthodes issues de disciplines différentes mais complémentaires (épidémiologie, sciences sociales, sciences politiques, sciences économiques...). Cette diversité d'approches quantitatives, qualitatives, critiques, mixtes permet une compréhension plus complète du problème étudié, la validation/ infirmation de la totalité ou d'une partie des résultats, l'illustration du contexte, l'examen des processus et/ou des expériences au sein de l'intervention [19]. Cette posture méthodologique plurielle doitêtre intégrée dès le processus de formation à la recherche en santé publique.

En parallèle, une action de plaidoyer est nécessaire auprès des décideurs et financeurs qui raisonnent sur les modèles classiques d'évaluation de santé publique, et les appels à projet doivent permettre de présenter dès la conception d'un projet comment cette diversité de méthodes sera utilisée comme un atout pour le développement et les évaluations des interventions complexes.

\section{Perspectives}

Les échanges du 2 juin 2014 entre chercheurs et financeurs d'interventions complexes en santé des populations ont replacé au centre du débat le besoin partagé de mieux préciser la conception, la définition et l'évaluation d'une intervention complexe dans le domaine de la santé publique en France. Les pistes de réflexions engagent à la fois les chercheurs qui conduisent ces programmes et les structures de financement qui participent, par leurs appels à projet, au développement età l'orientation de la capacité de recherche. Les propositions qui émanent de cette rencontre sontissues des invariants trouvés dans les différentes présentations faites par les participants de cette réunion lesquelles se sont aussi largement inspirées des débats en cours dans différents pays. En effet, la littérature académique comporte plusieurs exemples d'application de ce programme d'action sur les DSS notamment au Canada, aux USA et en Europe [20-22].

Cette synthèse des échanges et les cinq axes de plaidoyer qui peuvent être adressés aux décideurs, montrent, comme Howden-Chapman [23] l'avait déjà exprimé, que la mise en œuvre du programme prenant en compte les DSS dans la pratique, a besoin d'un fort soutien public, d'approches collectives stratégiques, et d'évaluations rigoureuses des interventions pour pouvoir contribuer à ce que les décideurs mettent en œuvre des politiques efficaces. Ces conclusions ne sont donc ni nouvelles ni uniques. Toutefois, aujourd'hui, les pistes de réflexions présentées ici, issues d'un état des lieux du contexte français, soulignent que ces défis sont partagés avec la communauté internationale, et que chercheurs, financeurs et acteurs français doivent dès demain s'engager dans des concertations devenues nécessaires en termes de recherche interventionnelle, d'évaluation et d'implémentation des interventions complexes en santé publique.

Aucun conflit d'intérêt déclaré

\section{Références}

1. Breton $E$, Porcherie M. La pratique en promotion de la santé en France. La santé de l'Homme. 2012;420:57-58. 
2. Société Française de Santé Publique (SFSP). Résultats de la consultation ouverte sur les inégalités sociales de santé. Laxou : SFSP ; 2014. 58 p. [Visité le 28/10/2015]. En ligne : http://www.sfsp.fr/activites/file/RapportSFSPCSISSjuin2014.pdf.

3. Hawe P, Potvin L. What is population health intervention research? Can J Public Health. 2009;100(1):18-114.

4. Organisation mondiale de la santé(OMS), Commission des déterminants sociaux de la santé. Combler le fossé en une génération: instaurer l'équité en santé en agissant sur les déterminants sociaux de la santé : rapport final de la Commission des Déterminants Sociaux de la Santé. Genève: OMS; 2008. 40 p. [Visité le 28/10/2015]. En ligne: http://apps.who.int/iris/ bitstream/10665/69831/1/WHO_IER_CSDH_08.1_fre.pdf.

5. Datta J, Petticrew M. Challenges to evaluating complex interventions: a content analysis of published papers. BMC Public Health. 2013;13:568. doi : 10.1186/1471-2458-13-568.

6. Craig P, Dieppe P, Macintyre S, Michie S, Nazareth I, Petticrew M. Developing and evaluating complex interventions : new guidance. Londres: Medical research council; 2008. 39 p. [Visité le 28/10/2015]. En ligne: http://www.mrc.ac.uk/complex interventionsguidance.

7. Wang S, Moss JR, Hiller JE. Applicability and transferability of interventions in evidence-based public health. Health Promot Int. 2006; 21(1):76-83.

8. Cambon L, Ridde V, Alla F. Réflexions et perspectives concernant l'evidence-based health promotion dans le contexte français. Rev Epidemiol Santé Publique. 2010;58(4):277-83.

9. Tarquinio C, Kivits J, Minary L, Coste J, Alla F. Evaluating complex interventions: perspectives and issues for health behaviour change interventions. Psychol Health. 2015;30(1):35-51.

10. Trickett E, Beehler S, Deutsch C, Green L, Hawe P, McLeroy K, et al. Advancing the science of community level interventions. Am J Public Health. 2011;101(8):1440-19.

11. Braveman P, Egerter S, Williams DR. The social determinants of health: Coming of Age. Annu Rev Public Health. 2011;32:381-98.

12. McLaren L, Hawe P. Ecological perspectives in health research. J Epidemiol Community Health. 2005;59(1):6-14.
13. Williams G, Elliot E. Exploring social inequalities in health: the importance of thinking qualitatively. In: Bourgeault I, Dingwall $R$, De Vries R, éditeurs. The SAGE handbook qualitative methods health

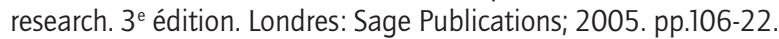

14. Pawson R. The science of evaluation: a realist manifesto. Londres: Sage Publications; 2013. 240 p.

15. Bonner L. Using theory-based evaluation to build evidence-based health and social care policy and practice. Critical Public Health. 2013;13(1):77-92. doi : 10.1080/0958159031000100224.

16. Manzano-Santaella A. A realistic evaluation of fines for hospital discharges: Incorporating the history of programme evaluations in the analysis. Evaluation. 2011;17(1):21-36.

17. Glasgow RE, Lichtenstein $E$, Marcus AC. Why don't we see more translation of health promotion research to practice? Rethinking the efficacy-to-effectiveness transition. Am J Public Health. 2003;93(8):1261-7.

18. Cambon L, Minary L, Ridde V, Alla F. Transferability of interventions in health education: a review. BMC Public Health. 2012;12:497. doi : 10.1186/1471-2458-12-497.

19. Plano Clark VL. The adoption and practice of mixed methods: U.S. trends in federally funded health-related research. Qualitative Inquiry. 2010;16(6):428-40.

20. Cahuas MC, Wakefield S, Peng Y. Social change or business as usual at city hall? Examining an urban municipal government's response to neighbourhood-level health inequities. Soc Sci Med. 2015;133:366-73.

21. Weber L, Hilfinger K. Mississippi front-line recovery work after Hurricane Katrina: An analysis of the intersections of gender, race, and class in advocacy, power relations, and health. Soc Sci Med. 2011;74(11):1833-41.

22. Eriksson M, Emmelin M. What constitutes a health-enabling neighborhood? A grounded theory situational analysis addressing the significance of social capital and gender. Soc Sci Med. 2013;97:112-23.

23. Howden-Chapman E. Evidence-based politics: How successful have government reviews been as policy instruments to reduce health inequalities in England? Soc Sci Med. 2010;71(7):1240-3. 